\title{
Large-scale Volunteer Engagement in Humanitarian Mapping
}

Martin Dittus

ICRI Cities, UCL

London, UK

martin@dekstop.de
Permission to make digital or hard copies of part or all of this work for personal or classroom use is granted without fee provided that copies are not made or distributed for profit or commercial advantage and that copies bear this notice and the full citation on the first page. Copyrights for third-party components of this work must be honored. For all other uses, contact the Owner/Author. Copyright is held by the owner/author. CSCW'16 Companion, February 27-March 02, 2016, San Francisco, CA, USA. ACM 978-1-4503-3950-6/16/02. DOI: http://dx.doi.org/10.1145/2818052.2874351

\begin{abstract}
Organisers of large crowdsourcing initiatives need to consider how to produce outcomes with their projects, but also how to build volunteer capacity. The initial contributor experience plays an important role in this, particularly when contributions require some expertise: not all contributors who start to learn the practice are likely to be retained. My dissertation is focused on this growth challenge, using the example of the volunteer community of the Humanitarian OpenStreetMap Team. The research involves several quantitative observational studies at the scale of the individual, the group, and the collective, and qualitative studies of contributor experiences.
\end{abstract}

\section{Author Keywords}

Crowdsourcing; Peer Production; Social Computing; Task Design; Social Presence; Engagement; Retention.

\section{ACM Classification Keywords}

H.5.3. [Group and Organization Interfaces]: Computersupported cooperative work

\section{Introduction}

It may be taken for granted that the maps used to navigate our urban spaces will be just as useful in other parts of the world, however the reality is different: when humanitarian aid teams attempted to follow the Ebola outbreak 


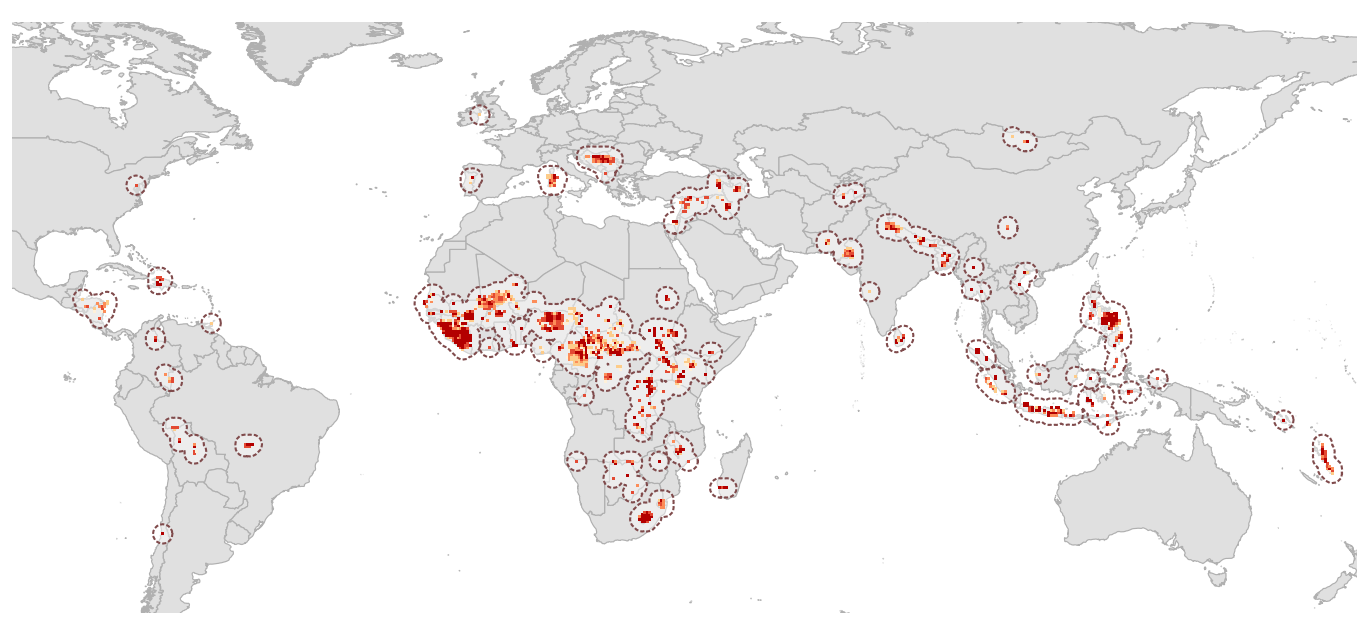

Figure 1: Geographic distribution of all HOT contributions.

in summer 2014 they found that most affected settlements were on no existing map [4]. In response, the Humanitarian OpenStreetMap Team (HOT) coordinated thousands of volunteers to trace roads, waterways, huts and houses from satellite data. In some cases, regional groups hosted mapathons to come together in a more social setting, but many contributors simply participated online.

HOT first started as an informal network of experts and community organisers to create free, up-to-date maps for humanitarian purposes, and the organisation has gradually refined the necessary processes and technologies that allow it to scale [6]. It coordinated responses to typhoon Haiyan in 2013, the West African Ebola crisis in 2014, the $2015 \mathrm{Nepal}$ earthquake, and many others [7]. Figure 1 visualises the geographic distribution of HOT map contributions to date.
The formidable scale of their ambition was illustrated during the 2014 Ebola epidemic: even after months of work by thousands of volunteers, the new maps of Central and West Africa are still not complete. An article by Médecins Sans Frontières suggests that to reach their goal, HOT organisers need to grow their project to "the biggest instance of digital volunteerism the world has ever seen" [3]. Organisers thus not only need to consider how to produce these maps, but also how to foster a large global volunteer community in the process.

My research is focused on this growth challenge: to develop understanding of how best to increase volunteer capacity. It takes the form of several large-scale quantitative observational studies, and includes qualitative studies of the contributor experience.

\section{Research Questions}

Research of contributor engagement in a crowdsourcing system requires the consideration of a complex range of socio-technical aspects. HOT activities involve an evergrowing set of systems and practices. Furthermore, there are likely many interactions between causal factors. In an effort to manage this complexity, we propose to regard the volunteer growth challenge at three complementary scales: task design and contribution mechanics (individual experience), social contribution settings involving peer learning (group experience), and the interplay of larger mapping initiatives over longer periods (collective experience).

\section{Task Design}

A first study in summer 2015 was concerned with the microfoundations of contributor engagement: task design and contribution mechanics. Are different task designs associated with different engagement characteristics? Tasks that overwhelm can be an impediment to early mastery 
experiences, a prerequisite for building contributor selfefficacy [1]. HOT projects were classified across multiple task complexity dimensions, grounded in existing theory and refined in a iterative process. This allowed to then as sess the impact that contribution mechanics can have on initial contributor engagement.

\section{Mapathons}

During a mapathon, HOT newcomers and experienced contributors come together in a social setting to learn the practice. An ongoing study of a range of different mapathon events seeks to assess their impact on subsequent contributor engagement. How successful are mapathons at training new contributors? Do certain kinds of settings foster more engaged contributor communities? How important is the social setting, for example the presence of experienced mappers? What other factors do organisers need to consider when they plan events?

\section{Contributor Flows}

A third upcoming study will identify larger mapping initiatives, observe the flow of contributors across them, and assess their mutual relationships. Do initiatives benefit from each others' activities through cross-promotion and other practices, or do they compete for attention? What contributor flows can be observed during times of large disaster events? Are there forms of preferential attachment, or do mapping activities follow highly promoted needs? How can previous contributors be reactivated during disasters?

\section{Work in Progress}

A first study assessed the impact of task design and other coordination practices on contributor engagement. 1,570 contributors across three of the largest HOT initiatives were observed over an 18-month period, covering 99 HOT projects. This represents a large subset of all HOT map- ping activity to date. The results have already been published [2].

Study findings suggest that task designs can be a deterrent for certain contributor groups: more complex task requirements may be demotivating to first-time contributors, regardless of their prior domain experience. In other respects, the task designs encountered in our study were found to be remarkably consistent in their engagement characteristics. Many of these designs have already been informed by years of prior experience [5].

It was further found that coordination practices may have a larger impact on contributor engagement than current task design choices. HOT initiatives with the highest contributor retention rates employed a combination of practices: sustained promotion, a steady stream of new efforts, regular mapathons and other training environments, and the use of email notifications to pull contributors into new projects.

A follow-up study of mapathon engagement is now in progress. The engagement trajectory of first-time attendees is compared across a range of different mapathons, some open to the public, some organised for members of large organisations. Three phases of participation are distinguished: the initial learning of the contribution practice during the event (initiation), subsequent mapping at home over the following days (activation), and contributions to HOT projects over longer periods (retention, or membership).

Early analyses show that most attendees are successfully initiated, however that many are not activated in the following days: they do not continue to map at home. Those that are activated tend to then remain engaged over longer periods. Upcoming research will seek to identify factors that can improve the rate of activations. 
Research findings to date suggest that sustained community engagement is only partially a matter of optimising the contribution process. In addition, factors relating to the individual and their participation context are likely just as important. Anecdotally, highly engaged mapathon attendees are often GIS specialists, volunteers for aid organisations, or otherwise embedded within existing contributor communities. In contrast, online contributors who join HOT during a widely publicised emergency response are unlikely to be retained. It appears unlikely that prolific mappers are "created" by a particular process, but they can be discovered, and they likely need some support in order to get started.

\section{Expected Contributions}

An immediate outcome of this work is a better understanding of the HOT contributor engagement trajectory, the identification of some critical factors in this process, advice for organisers across a range of concerns, and suggestions for more detailed follow-up studies. The range of existing HOT initiatives and organisational practices offers many opportunities to evaluate specific organiser choices based on readily available public data.

This research is further adding to a growing body of knowledge about how to engage and retain large numbers of volunteers in crowdsourcing systems. This includes insights about where best to spend limited organiser resources, and how to reconcile the dual aim of producing high-quality outcomes while building a large volunteer community.

The work involves the development of computational methods that are grounded in existing theory and yield operational insights from readily available public data. The methods provide minimum-effort complements to more invasive evaluation practices such as controlled experiments, $A / B$ tests and participant observations. They are transferrable to other settings: their minimum requirement is a capacity to observe individual contributions over time.

\section{Acknowledgments}

This research is funded by the EPSRC and the Intel Collaborative Research Institute: Cities. I am grateful to Andrew Braye, Pete Masters, and countless others in the HOT community for their support, and to my supervisor Licia Capra for her invaluable feedback and guidance.

\section{References}

[1] Albert Bandura. 1977. Self-efficacy: toward a unifying theory of behavioral change. Psychological review 84, 2 (1977), 191.

[2] Martin Dittus, Giovanni Quattrone, and Licia Capra. 2016. Analysing Volunteer Engagement in Humanitarian Mapping: Building Contributor Communities at Large Scale. In Proc. CSCW'16.

[3] Médecins Sans Frontières. 2014. MSF: the Missing Maps project. (2014). http://www.msf.org.uk/ missing-maps-project.

[4] Chris Michael. 2014. Missing Maps: nothing less than a human genome project for cities - The Guardian. http://www.theguardian.com/cities/2014/oct/ 06/missing-maps-human-genome-project-unmapped-cities. (2014).

[5] Leysia Palen, Robert Soden, T Jennings Anderson, and Mario Barrenechea. 2015. Success \& scale in a data-producing organization: the socio-technical evolution of OpenStreetMap in response to humanitarian events. In Proc. SIGCHI '15. 4113-4122.

[6] Robert Soden and Leysia Palen. 2014. From crowdsourced mapping to community mapping: the postearthquake work of OpenStreetMap Haiti. (2014).

[7] Humanitarian OpenStreetMap Team. 2015. Featured HOT projects. http://hotosm.org/projects. (2015). 